Article

\title{
Simvastatin Attenuates the Oxidative Stress, Endothelial Thrombogenicity and the Inducibility of Atrial Fibrillation in a Rat Model of Ischemic Heart Failure
}

\author{
Kyoung-Im Cho, Sang-Ho Koo, Tae-Joon Cha *, Jung-Ho Heo, Hyun-Su Kim, Gee-Bum Jo \\ and Jae-Woo Lee \\ Division of Cardiology, Department of Internal Medicine, Kosin University College of Medicine, \\ Busan 602702, Korea; E-Mails: kyoungim74@gmail.com (K.-I.C.); paulkoo@hanmail.net (S.-H.K.); \\ lduggymdc@gmail.com (J.-H.H.); libaek@gmail.com (H.-S.K.); kyoungim74@dreamwiz.com (G.-B.J.); \\ jwlee@ns.kosinmed.or.kr (J.-W.L.) \\ * Author to whom correspondence should be addressed; E-Mail: chatjn@gmail.com; \\ Tel.: +82-51-990-6725; Fax: +82-51-990-3047.
}

Received: 13 May 2014; in revised form: 21 July 2014 / Accepted: 1 August 2014 /

Published: 22 August 2014

\begin{abstract}
Increased atrial oxidative stress has an important role in inducing and maintaining atrial fibrillation (AF), and the activation of the small GTPase Rac1 contributes to the oxidative stress. We investigated the relationship of Rac1, atrial endothelial thromboprotective markers and AF inducibility and if simvastatin has a potential beneficial effect on a myocardial infarction (MI)-induced heart failure (HF) rat model. Rats were randomized into three groups (shams, MI group and simvastatin treatment group) and underwent echocardiography, AF induction studies and left atrial (LA) fibrosis analysis. Atrial Rac 1, sodium calcium exchanger $\left(I_{\mathrm{NCX}}\right)$, sarcoplasmic reticulum calcium ATPase (SERCA), endothelial nitric oxide synthase (eNOS) and induced nitric oxide synthase (iNOS) were measured. AF inducibility, AF duration and LA fibrosis were significantly higher in the MI group $(p<0.001 v s$. sham), which were significantly reduced by simvastatin ( $p<0.05 v s$. MI). The reduced expressions of atrial eNOS, SERCA, thrombomodulin, tissue factor pathway inhibitor and tissue plasminogen activator in the MI group were significantly improved by simvastatin. Furthermore, the increased expression of atrial iNOS, $I_{\mathrm{NCX}}$ and Rac1 activity were significantly decreased by the simvastatin. Oxidative stress, endothelial dysfunction and thrombogenicity
\end{abstract}


are associated with the promotion of AF in a rat model of ischemic HF. These were associated with increased Rac1 activity, and simvastatin treatment prevents these changes.

Keywords: statin; atrial fibrillation; nitric oxide synthase; heart failure

\section{Introduction}

Atrial fibrillation (AF) is the most common clinically significant cardiac arrhythmia and is a potent risk factor for ischemic stroke [1]. Recent studies have suggested that inflammation and oxidative stress play a role in the pathogenesis of AF [2-5]. Because hypercholesterolemia reduces endothelial production and increases degradation of nitric oxide (NO) [6,7], cholesterol-lowering treatment by statins causes a significant improvement in endothelial function [6] by upregulating endothelial cell nitric oxide synthase (eNOS) and NO synthase [8]. Some studies have suggested that statins block atrial remodeling induced by substances such as NO and reactive oxygen species [9,10]. In addition to their indirect antiarrhythmic effects, statins may also exhibit direct antiarrhythmic effects by modulating the fatty acid composition and physiochemical properties of cell membranes, with resultant alterations in transmembrane ion channel properties [10,11]. Moreover, accumulating evidence suggests that statins may reduce vascular inflammation and the incidence of AF [12] by virtue of pleiotropic properties independent of lipid lowering and that statins may significantly reduce the incidence of AF in the setting of both primary and secondary prevention $[13,14]$. According to Sarr et al. [15], hydrophilic pravastatin exhibited the lowest association with the lipid monolayer, and lipophilic simvastatin showed a strong membrane elution ability, which can be explained by the hydrophobicity of the statin molecule [16]. These findings suggest that the difference of membrane permeability according to the lipophilicity might explain the impact of statin on the ion channel in the cell membrane.

Observations of ventricular cardiomyocytes in heart failure (HF) have revealed that the action potential duration is prolonged and that sarcoplasmic reticulum $\mathrm{Ca}^{2+}$ release is reduced under these conditions [17]. Abnormal intracellular $\mathrm{Ca}^{2+}$ cycling plays an important role in cardiac dysfunction and ventricular arrhythmogenesis in the setting of ischemic HF [18,19]. However, the antiarrhythmic effects of statins in ischemic HF are still unknown.

Atrial oxidative stress might play an important role in inducing and maintaining $\mathrm{AF}$, and nicotinamide adenine dinucleotide phosphate (NADPH) oxidase activity is a major source of myocardial superoxide production [20]. Rac1 is necessary for the activation of the NADPH oxidase complex, and during activation, Rac1 binds guanosine triphosphate (GTP) and migrates to the membrane with the core cytosolic complex. Therefore, it has been suggested that the activation of Rac1 GTPase might contribute to the pathogenesis of AF via activation of superoxide producing NADPH oxidase [21].

We hypothesized that there would be the relationship between Rac 1 and AF inducibility, as well as atrial endothelial thromboprotective markers, which might be reduced by statin. Then, we hypothesized that the altered $\mathrm{Ca}^{2+}$ handling, inflammation and thromboprotective markers in ischemic HF are attenuated by the statin, which might explain the anti-arrhythmic role of statin in HF. Therefore, we investigated 
the changes in Rac 1, the inducibility of AF and atrial endothelial thromboprotective markers with or without statin in a rat model of ischemic HF model.

\section{Results and Discussion}

\subsection{Echocardiographic Indices}

Echocardiographic parameter changes in each group are presented in Figure 1 and Table 1. In the myocardial infarction (MI) group $(n=10)$, the left ventricular end diastolic dimension and left atrial (LA) dimension were significantly increased and the ejection fraction significantly decreased $(30 \% \pm 1.6 \%$ vs. $83 \% \pm 7.5 \%, p<0.001)$ compared to the sham group $(n=10)$ (Figure 1, Table 1).

Figure 1. Echocardiograms exhibiting the left ventricular anteroseptal wall akinesia and dilated ventricular dimensions in myocardial infarction (MI) rats. These changes were slightly less pronounced in the simvastatin-treated group. IVS, interventricular septum; LV, left ventricle; LVPW, left ventricular posterior wall.

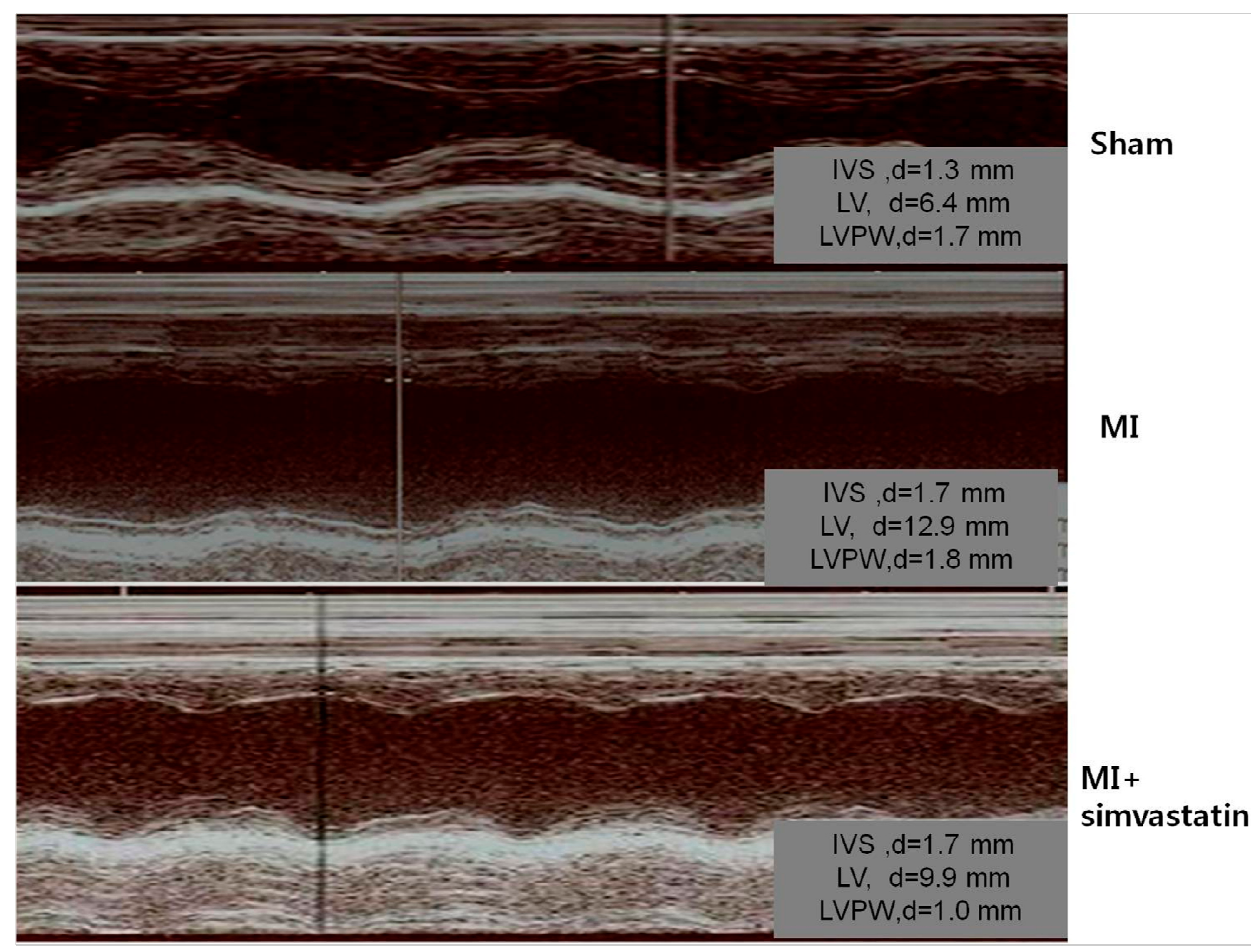

Table 1. Echocardiographic parameters.

\begin{tabular}{cccc}
\hline Parameters & Sham $(\boldsymbol{n}=\mathbf{1 0})$ & MI $(\boldsymbol{n}=\mathbf{1 0})$ & MI-Simvastatin $(\boldsymbol{n}=\mathbf{1 0})$ \\
\hline EF, \% & $83.0 \pm 7.6$ & $30.0 \pm 16.1 *$ & $43.0 \pm 21.5^{*}$ \\
FS, \% & $48.5 \pm 8.8$ & $12.7 \pm 7.5 *$ & $20.6 \pm 15.2 *$ \\
LVEDD, mm & $7.9 \pm 0.6$ & $9.5 \pm 1.3^{* *}$ & $9.6 \pm 1.6 * * *$ \\
LVESD, mm & $4.0 \pm 0.8$ & $8.3 \pm 1.5 *$ & $7.8 \pm 2.2 *$ \\
LAD, mm & $3.4 \pm 0.8$ & $3.8 \pm 0.9$ & $3.6 \pm 0.8$ \\
\hline
\end{tabular}

$* p<0.001$ vs. sham; ** $p<0.01$ vs. sham; *** $p<0.05$ vs. sham; MI, myocardial infarction; EF, ejection fraction; FS, fractional shortening; LVEDD, left ventricular end diastolic dimension; LVESD, left ventricular end systolic dimension; LAD, left atrial dimension. 
There was a significant increase in the heart weight in the MI group compared to the sham group $(5.60 \pm 0.22$ vs. $3.37 \pm 0.13 \mathrm{mg}, p<0.001)$. The $\mathrm{MI}+\operatorname{simvastatin}$ group $(n=10)$ showed an increase in the ejection fraction (EF) $(43.2 \% \pm 2.2 \%$ vs. $30 \% \pm 16 \%, p=0.08)$ when compared to the MI group, but the difference was not statistically significant.

\subsection{Atrial Fibrillation Induction Study}

AF was induced more easily $(40.4 \% \pm 4.2 \%$ vs. $1.0 \% \pm 3.2 \%, p<0.001)$ in the MI group $(n=10)$ compared to the sham group $(n=10)$, but decreased after simvastatin treatment $(n=10,9.3 \% \pm 7.8 \%$ in the MI + simvastatin group, $p<0.05$ vs. MI) (Figures 2 and 3A). The duration of AF was also significantly increased in the MI group compared to the sham group (907 $\pm 942 v s .3 .0 \pm 9.5 \mathrm{~s}, p<0.001)$, but was moderately reduced after simvastatin treatment $(184 \pm 568 \mathrm{~s}$ in the $\mathrm{MI}+$ simvastatin group, $p<0.05$ vs. MI) (Figure 3B).

Figure 2. The electrocardiography shows the successful induction of atrial fibrillation (AF) following burst pacing in the myocardial infarction (MI group) (A); AF could not be induced in the sham group (B); and the duration of AF was lower in the MI + simvastatin group $(\mathbf{C})$.
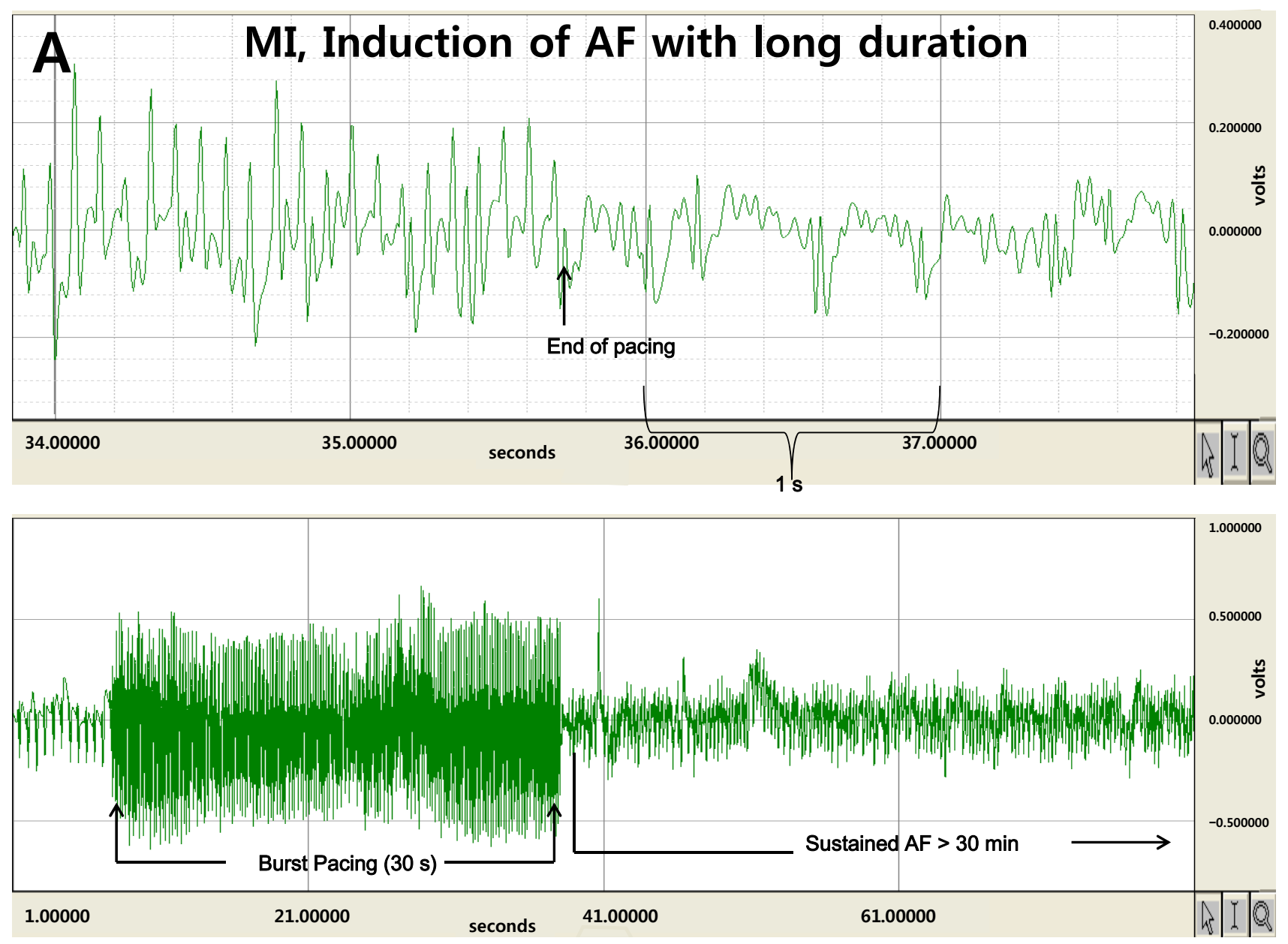
Figure 2. Cont.

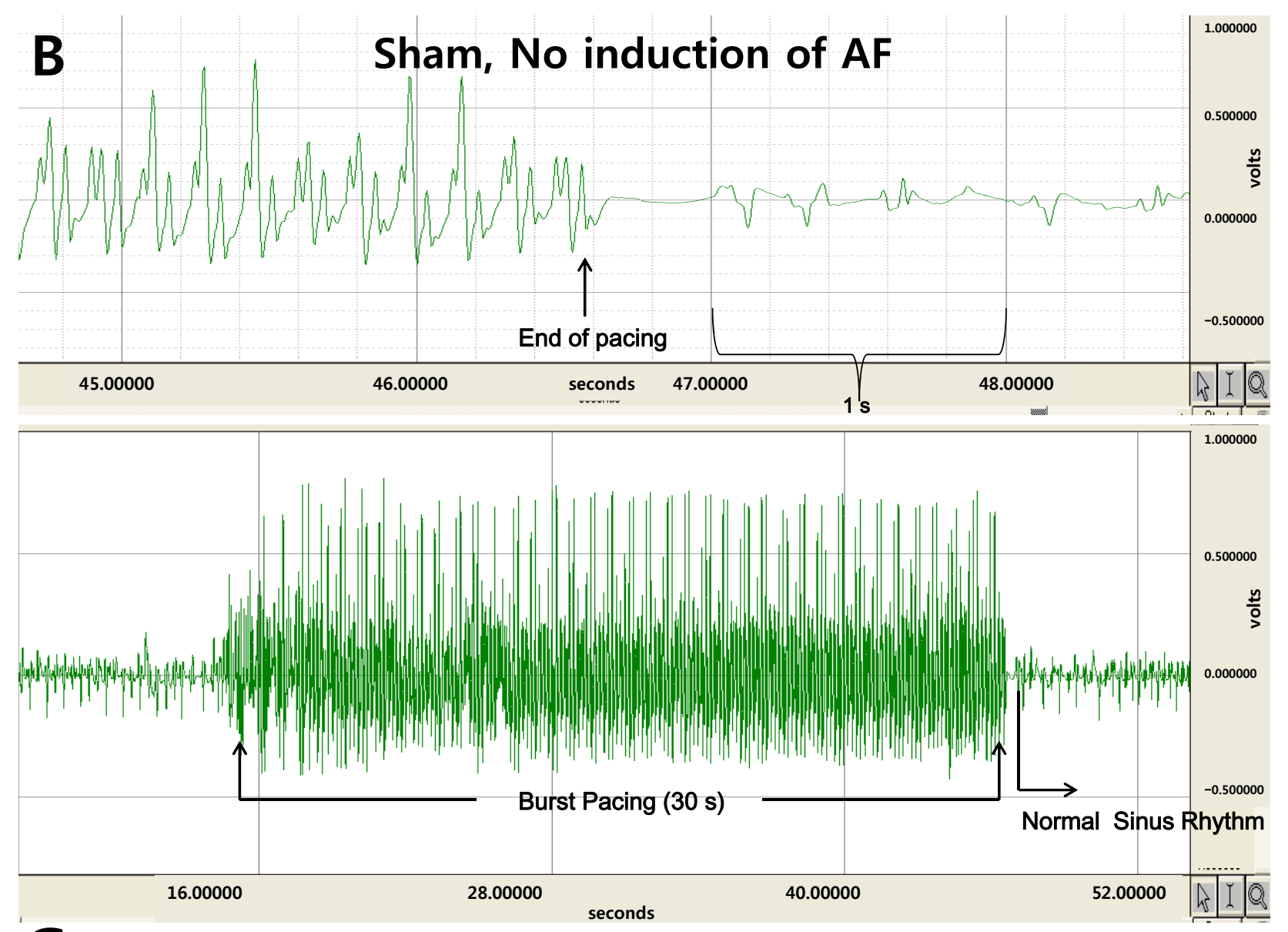

MI+simvastatin, temporary AF

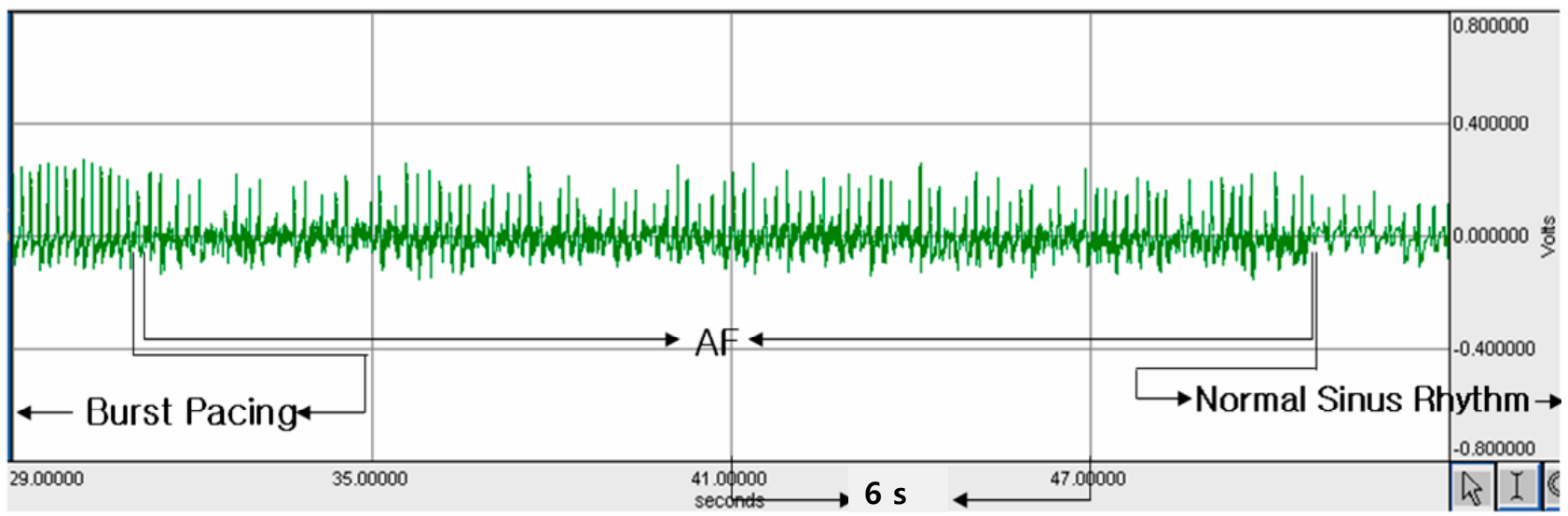

\subsection{Reduction of Atrial Fibrosis by Simvastatin}

The amount of fibrosis in the LA, as a percentage of perimuscular interstitial blue area except muscle for each rat, was significantly higher in the MI group than in the sham group $(n=7$ for each group, $2.30 \% \pm 0.71 \%$ vs. $0.24 \% \pm 0.09 \%, p=0.001$ ) (Figure 4). The amount of fibrosis in the LA was significantly lower in the MI + simvastatin group than in the MI group $(1.17 \% \pm 0.16 \%$ vs. $2.30 \% \pm 0.71 \%$, $p=0.001)$. 
Figure 3. (A) The results of the atrial fibrillation (AF) induction studies. ${ }^{*} p<0.001$ sham vs. myocardial infarction (MI), ${ }^{*} p=0.023$ MI vs. MI + simvastatin; and (B) The mean duration of $\mathrm{AF}$ (seconds) following burst pacing. $* p=0.001$ sham vs. $\mathrm{MI}$ and $\mathrm{MI}+$ simvastatin, ${ }^{\#} p=0.031 \mathrm{MI} v s . \mathrm{MI}+$ simvastatin.
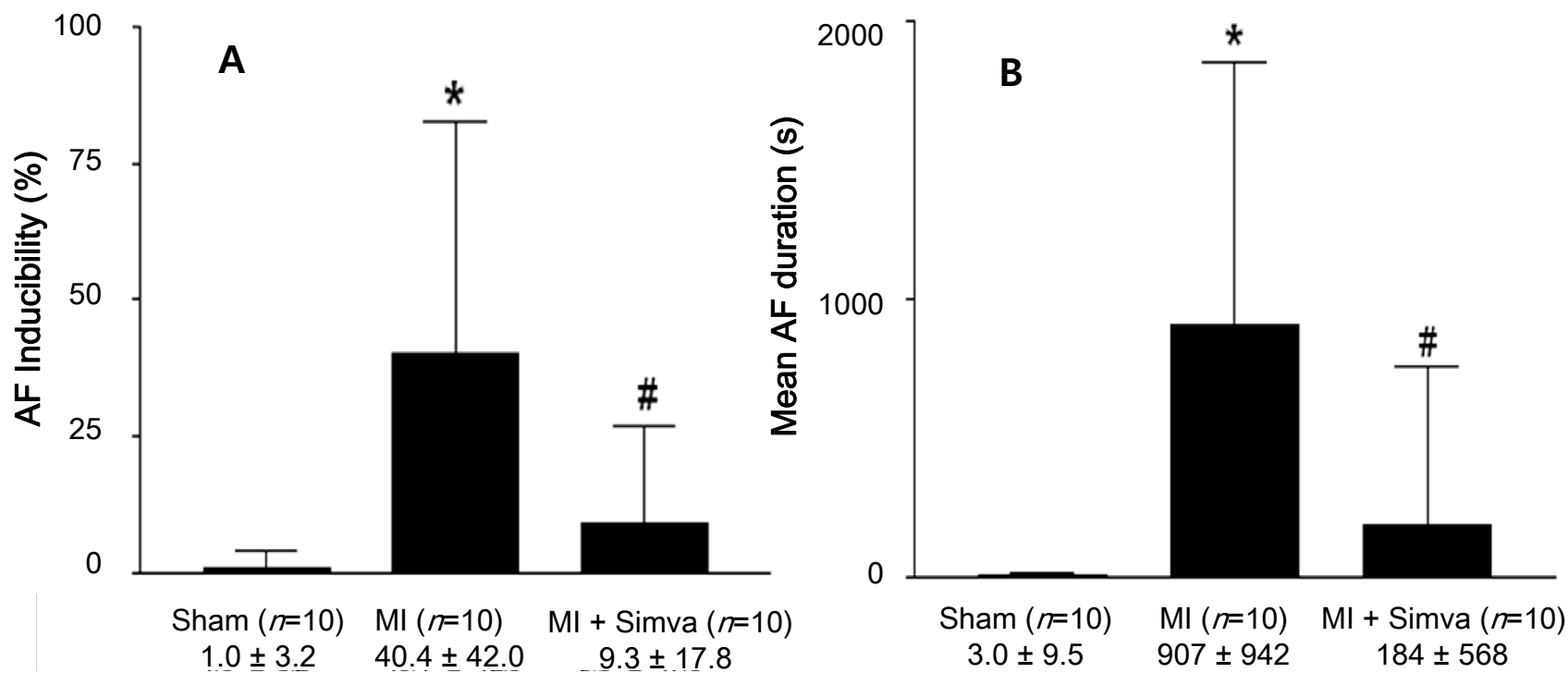

Figure 4. Masson's trichrome staining of the left atrium. The amount of fibrosis was lower in the sham (A) than the MI group (B); but this increase was attenuated in the MI + simvastatin (C) group. Blue represents fibrosis. (D) The effect of simvastatin on fibrosis of the left atrium. ${ }^{*} p=0.001$ sham $v$. MI and MI $+\operatorname{simvastatin}^{*}{ }^{*} p=0.001 \mathrm{MI}$ vs. MI + simvastatin.
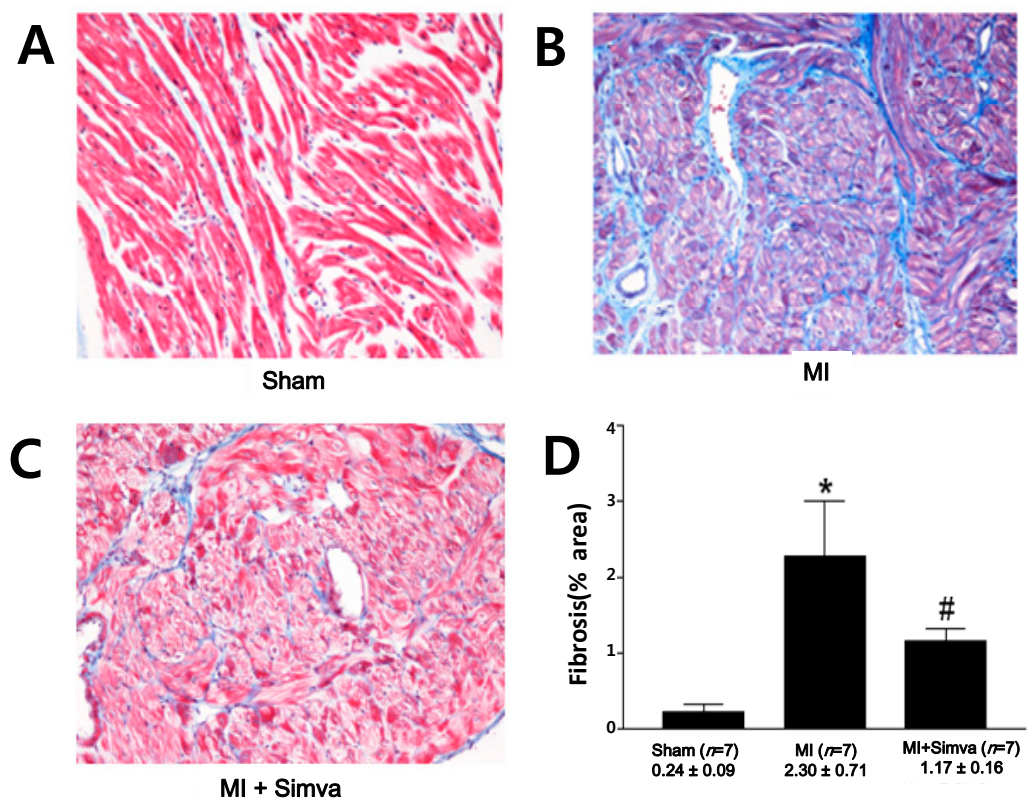

\subsection{Expression of Nitric Oxide Synthases and Calcium Handling Proteins}

LA tissue levels of the eNOS $(p<0.05)$ and SERCA $(p<0.01)$ proteins were significantly lower in the MI group than in the sham group, but were significantly higher in the MI + simvastatin group than in the MI group (all $p<0.05$, Figure $5 \mathrm{~A}$ ). The levels of iNOS and $I_{\mathrm{NCX}}$ proteins in LA tissue were 
significantly higher in the MI group than in the sham group $(p<0.01)$. The expression of iNOS and $I_{\mathrm{NCX}}$ was significantly lower in the $\mathrm{MI}+$ simvastatin group than in the MI group $(p<0.05)$ (Figures $5 \mathrm{~A}$ and Table 2). All results were normalized to GAPDH data concomitantly obtained from the same samples.

Figure 5. Alterations in eNOS, iNOS, SERCA, NCX (A), TFPI, TM, tPA (B) and Rac 1 (C) protein expression in the left atrium (eNOS, endothelial nitric oxide synthase; iNOS, induced nitric oxide synthase; NCX, sodium calcium exchanger; SERCA, sarcoplasmic reticulum calcium ATPase; TM, thrombomodulin; TFPI, tissue factor pathway inhibitor; tPA, tissue plasminogen activator; Rac 1, Rac 1 GTPase).

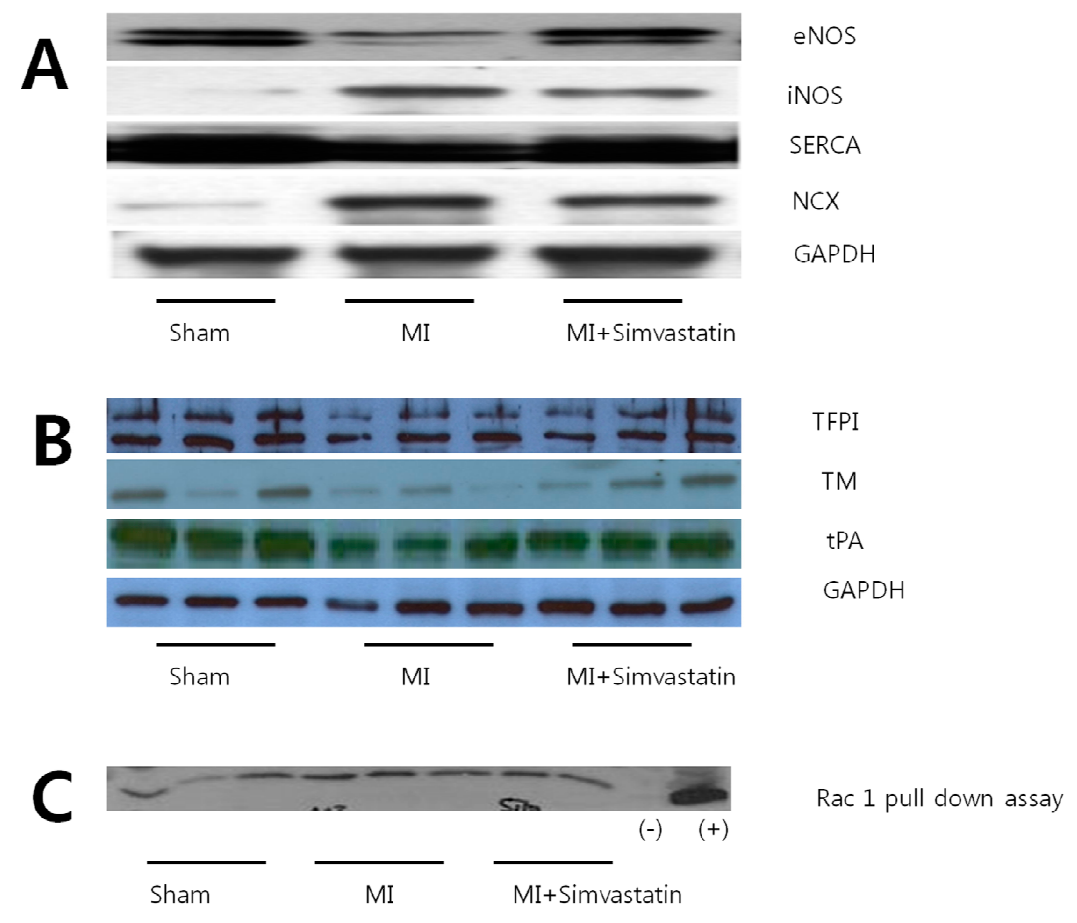

Table 2. Densitometric analysis of western blot results.

\begin{tabular}{cccc}
\hline Parameters & Sham $(\boldsymbol{n}=\mathbf{1 0})$ & MI $(\boldsymbol{n}=\mathbf{1 0})$ & MI-Simvastatin $(\boldsymbol{n}=\mathbf{1 0})$ \\
\hline Fibrosis $(\%)$ & $0.24 \pm 0.09(n=7)$ & $2.30 \pm 0.71 *(n=7)$ & $1.17 \pm 0.16^{\#}(n=7)$ \\
eNOS & $13.41 \pm 2.58(n=6)$ & $6.75 \pm 2.05 *(n=6)$ & $12.06 \pm 3.43^{\#}(n=6)$ \\
iNOS & $0.28 \pm 0.30(n=9)$ & $7.33 \pm 5.79 *(n=10)$ & $1.60 \pm 1.477^{* * \#}(n=12)$ \\
$I_{\text {NCX }}$ & $0.79 \pm 0.48(n=8)$ & $14.52 \pm 11.87 *(n=11)$ & $4.19 \pm 11.87 *^{* \# \#}(n=12)$ \\
SERCA & $30.90 \pm 3.18(n=6)$ & $21.13 \pm 1.36 *(n=6)$ & $25.43 \pm 3.27 * *$,\#\# $(n=6)$ \\
TM & $2.54 \pm 0.47(n=6)$ & $0.80 \pm 0.28 *(n=6)$ & $1.85 \pm 0.38^{\#}(n=6)$ \\
TFPI & $6.37 \pm 0.71(n=6)$ & $3.15 \pm 0.22(n=6)$ & $4.32 \pm 0.47(n=6)$ \\
tPA & $2.73 \pm 0.12(n=6)$ & $1.25 \pm 0.12(n=6)$ & $2.01 \pm 0.13(n=6)$ \\
Rac 1 & $16,165 \pm 1696(n=6)$ & $28,440 \pm 1655^{*}(n=6)$ & $19,275 \pm 2236^{\#}(n=5)$
\end{tabular}

$* p<0.01$ vs. sham, ** $p<0.05$ vs. sham; ${ }^{\#} p<0.001$ vs MI + simvastatin; \# $p<0.01$ vs. MI + simvastatin; eNOS, endothelial nitric oxide synthase; iNOS, inducible nitric oxide synthase; NCX, sodium calcium exchanger; SERCA, sarcoplasmic reticulum $\mathrm{Ca}^{2+}$-ATPase; TM, thrombomodulin; TFPI, tissue factor pathway inhibitor; tPA, tissue plasminogen activator; Rac 1, Rac 1 GTPase; values in the table are GAPDH-normalized, except Rac1 GTPase. 


\subsection{Immunohistochemistry Results}

Immunohistochemical staining for eNOS showed that most activity was located at the endocardial area in the sham group. Endocardial eNOS activity was lower in the MI group than in the control group, but the MI + simvastatin group had higher endocardial eNOS activity than the MI group (Figure 6A). Immunohistochemical staining for iNOS revealed little activity in the sham group, but myocardial iNOS activity was higher in the MI group. The MI + simvastatin group had lower iNOS activity in the myocardium than the MI group (Figure 6B).

Figure 6. (A) Immunohistochemical staining showed that eNOS expression in the MI group was lower than the sham, and eNOS expression in the MI + simvastatin group was higher than the MI group (endothelial line, red arrows); and (B) iNOS expression in the MI group was higher than the sham, and iNOS expression in the MI + simvastatin group was lower than the MI group (cytoplasmic brown area, black arrows).

\section{A enOS}
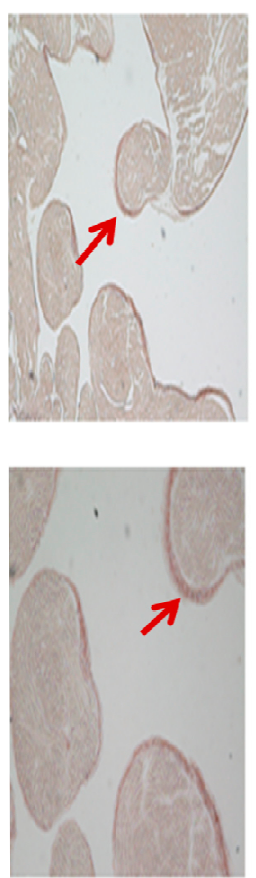

Sham
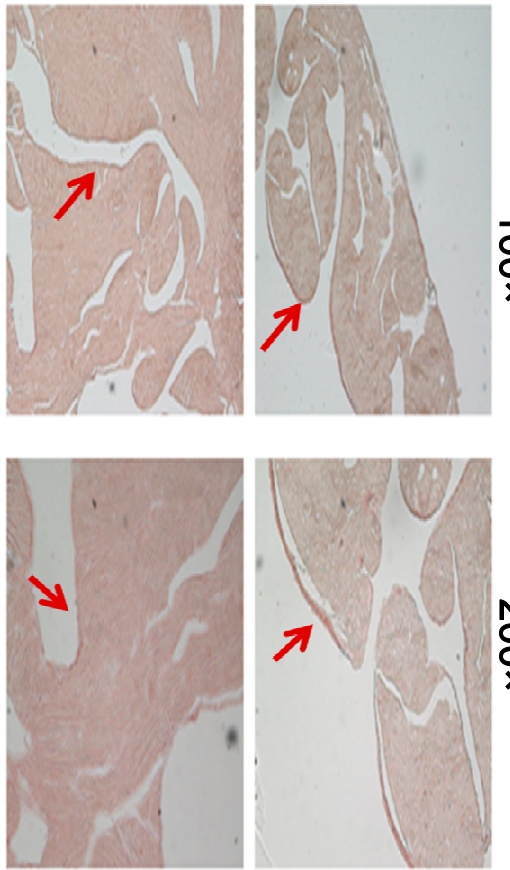

MI

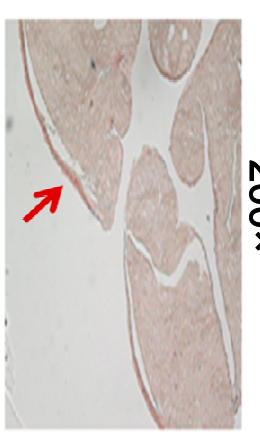

MI + Simva

\section{B inOS}
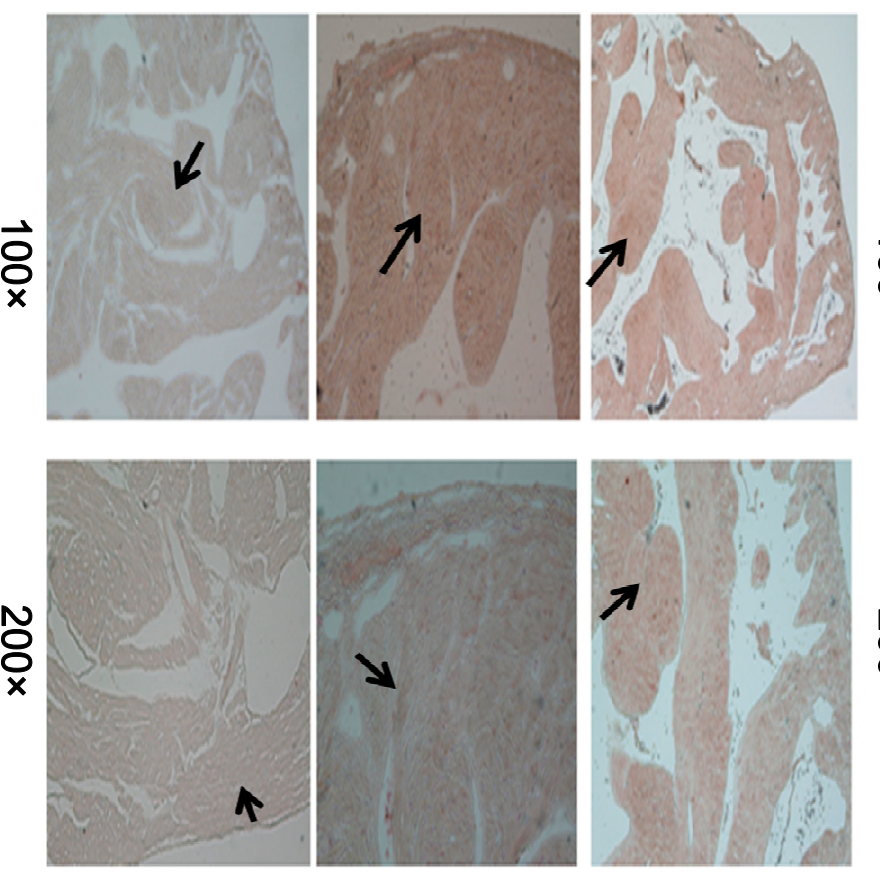

Sham

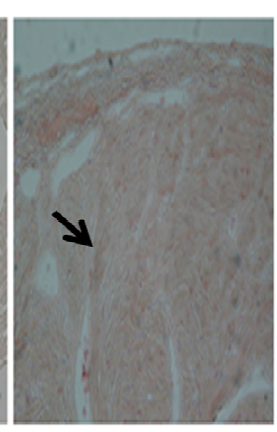

MI

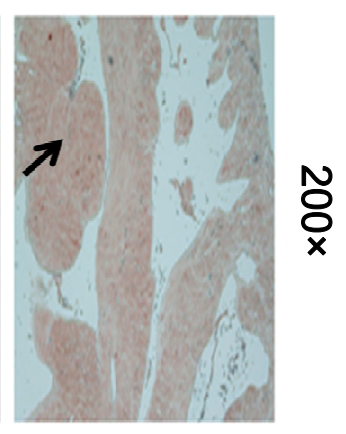

MI + Simva

\subsection{Thrombogenicity and Rac 1 Activity}

The levels of TM, TFPI, tPA and eNOS proteins in LA tissue were significantly decreased in the MI group than in the sham group $(p<0.05)$. These changes were improved in the MI + simvastatin group compared to the MI group $(p<0.05$ ) (Figure 5 and Table 2). All results were normalized to GAPDH data concomitantly obtained from the same samples. Cardiac Rac1 expression was strongly increased in the MI group, and the MI + simvastatin group showed significantly reduced Rac1 expression $(p<0.05)$ (Figure 5C and Table 2).

This study demonstrated increased Rac1, $I_{N C X}$ and iNOS expression, reduced SERCA and eNOS expression and activation of endothelial thromboprotective markers in a murine ischemic HF model. 
These findings were associated with an increased inducibility and duration of AF, and these changes were attenuated with simvastatin treatment, suggesting that the reduction of oxidative stress may be one of the mechanisms responsible for the antiarrhythmic effects of simvastatin in ischemic HF.

Recently, there have been several reports that have demonstrated an association between AF and the inflammatory process [2-5], and it has been suggested that statins may reduce vascular inflammation and the incidence of AF [12] due to antioxidant and anti-inflammatory effects [13,14]. Randomized clinical trials examining the efficacy of statins in HF (GISSI-HF and CORONA) did not show a benefit in mortality for patients with HF randomized to receive statins; however, the antiarrhythmic effects of statins in ischemic HF have not been investigated until now.

Our study addressed two mechanisms underlying the arrhythmias seen in ischemic HF. The first mechanism is reentry, which is associated with an increased amount of tissue fibrosis [22]. LA fibrosis is an important contributor to the AF seen in ischemic HF, and we found that simvastatin treatment decreased the LA fibrosis induced by the MI with which AF could be induced. Simvastatin also induced a regression of the cardiac hypertrophy and fibrosis and improved cardiac function in a transgenic rabbit model of human hypertrophic cardiomyopathy [23]. These results are consistent with the idea that a reduction in fibrosis may contribute to the antiarrhythmic effects of simvastatin. The other important mechanism of arrhythmias in HF is triggered activity [19], and Fenelon et al. [24] demonstrated that the majority of AF episodes had a focal mechanism by performing biatrial mapping in dogs with CHF. Simvastatin attenuated the propensity toward AF in the dog atrial tachycardia model [25], and it attenuated tachypacing-induced remodeling effects, including downregulation of the L-type $\mathrm{Ca}^{2+}$ channel alpha subunit expression and shortening of the effective refractory period (ERP).

NADPH oxidase activity is critical for generating oxidative stress, and activation of Rac1 GTPase might contribute to the pathogenesis of AF via activation of superoxide, producing NADPH oxidase [21]. Small GTPase Rac1 regulates certain subfamilies of NADPH oxidase, namely NOX1 and NOX2 [26,27]. NOX2 is upregulated only in patients with paroxysmal/persistent AF and is responsible for the overproduction of isoprostanes [28]. Statins block the isoprenylation and activation of small GTP-binding proteins members, such as the Ras and Rho family. An animal model has demonstrated that statins attenuate NADPH oxidase-induced superoxide production through the inhibition of Rac1 [29,30]. Based on these data, our result for the simvastatin-induced inhibition of Rac1 may decrease NOX2, which may reduce the risk of paroxysmal AF. However, in the case of permanent AF, this impact would be attenuated, because upregulation of atrial NADPH oxidases is an early, but transient event in the natural history of AF [30]. Moreover, our results showed that the increased Rac1 activity was associated with decreased atrial endothelial thromboprotective markers, as well as increased AF duration in ischemic HF rats. In addition to the inhibition of Rac1, the effect of statins on the isoprenylation of other proteins, as well as the anti-inflammatory effects of simvastatin is likely to contribute to the observed effects. Therefore, simvastatin might be useful in preventing thrombosis-related complications of AF.

Because changes in the NO synthase system were reported in a pig model of AF [4], the alteration in NO synthesis is thought to be an important contributor to AF-induced LA remodeling. In myocardial tissue, eNOS is constitutively expressed in the myocytes, endothelial cells and endocardial endothelium, while iNOS is induced after stimulation with lipopolysaccharide and cytokines in multiple cardiac cells, including myocytes, vascular endothelial and smooth muscle cells 
and inflammatory cells [31]. Because iNOS is expressed in pathological conditions, such as septic shock and HF [32], increased NO production in HF [33] is in part due to this increased iNOS and neuronal NO synthase (nNOS) expression [34]. In this study, we demonstrated decreased eNOS and increased iNOS expression in the LA tissue of the rat model of ischemic HF. Decreased eNOS is associated with an increased thrombogenicity in atrial tissue during HF, as evidenced by an increase in plasminogen activator inhibitor-1 (PAI-1) expression in the LA in a dog AF model [17]. These results support the idea that changes in eNOS expression contribute to tachypacing-induced atrial remodeling [4,32]. Interestingly, we also observed increased levels of iNOS in our study, which may also play a role in HF-induced LA remodeling in AF. A recent study demonstrated the relationship between superoxide and AF [35,36], and iNOS is associated with inflammation and superoxide synthesis. Increased iNOS suggests increased superoxide production in the LA tissue during HF, and statins have pleiotropic effects, including antioxidant and anti-inflammatory effects. In this study, we demonstrated that simvastatin prevented iNOS production in LA tissue during HF, and iNOS reduction may contribute to the antiarrhythmic effects of statins in HF.

This study also demonstrated increased production of $I_{\mathrm{NCX}}$ and decreased production of SERCA. $\mathrm{HF}$ increased the expression of $I_{\mathrm{NCX}}$, an important carrier of post-repolarization transient inward currents that cause delays after depolarizations (DADs). DADs can induce triggered activity, and there is evidence for the role of DADs in atrial tachyarrhythmias in canine CHF models [37,38]. Therefore, increased $I_{\mathrm{NCX}}$ expression in our study correlated with an increased susceptibility to AF in our rat model of $\mathrm{HF}$, and decreased $I_{\mathrm{NCX}}$ expression in simvastatin-treated rats is consistent with the concept of statins as anti-arrhythmic agents. SERCA, which plays a major role in the contraction-relaxation cycle [39], was reduced in our HF group. Reduced SERCA expression is also an important contributor to HF-induced LA remodeling, and SERCA expression was significantly higher in animals treated with simvastatin.

\section{Experimental Section}

\subsection{Animals Models}

Animal procedures were performed according to National Institutes of Health guidelines. The study protocol was approved by the Ethical Committee of Kosin University School of Medicine (Kosin 13-01, 6 February 2013). Seven- to eight-week-old healthy male Sprague-Dawley rats weighing 250-300 grams were obtained from the Daehan Biolink Inc. (Chungbuk, Korea). Animals were housed in plastic cages (five animals each) and fed with a normal sodium diet and given access to tap water. They were maintained under standard laboratory conditions (controlled temperature of $21-24{ }^{\circ} \mathrm{C}$, controlled light cycle consisting of alternating $12 \mathrm{~h}$ periods of light and darkness) during all experiments. An experimental rat model of post-MI HF was made from the ligation of the left anterior descending coronary artery (LAD), according to our previous study [37]. The rats were randomized into untreated MI and MI + simvastatin ( $2 \mathrm{mg} / \mathrm{kg}$ /day) groups after 2 days and observed for 10 weeks. The drugs were added to the drinking water, with careful monitoring of water consumption and body weight to ensure a proper drug dosage. Sham operated rats were used as a control group. The perioperative mortality was around $40 \%$ in the rats submitted to coronary artery ligation. The study was not financially 
funded by the pharmaceutical industry; however, simvastatin was generously provided by Merck Pharmaceuticals. Simvastatin was chosen because of the great lipophilicity, and it suppressed tachypacing-induced shortening of atrial refractoriness [25].

\subsection{Echocardiogram}

Echocardiography was performed in animals just before they were sacrificed. Animals were anesthetized by an intraperitoneal injection of ketamine $(25 \mathrm{mg} / \mathrm{kg})$ with xylazine $(5 \mathrm{mg} / \mathrm{kg})$. The chest was shaved, and the animals were placed on a warming pad to maintain normal body temperature. Transthoracic echocardiography was performed using a 12-MHz phased-array probe, Acuson Sequoia C512 (Mountain View, CA, USA). LV end-diastolic septal and posterior wall thickness (IVSd and PWTd, respectively), LV end diastolic dimension (LVEDD) and LV end systolic dimension (LVESD) were measured. The LV ejection fraction (EF) and fractional shortening (FS) were calculated according to the following formula: FS $(\%)=($ LVEDD - LVESD)/LVEDD, EF $(\%)=($ LVEDD2 LVESD2)/LVEDD2 $\times 100 \%$. Left atrial (LA) and aorta diameters were measured from the M-mode recordings in a modified parasternal long axis view.

\subsection{Electrophysiological Study}

The AF induction studies were performed in an open-chest state with a bipolar lead attached to the right atrial appendage. AF was induced by right atrial burst pacing (four times the pacing threshold for $30 \mathrm{~s}$ at $600 \mathrm{bpms}$ ). The mean duration of $\mathrm{AF}$ in each rat was determined on the basis of ten inductions. AF was induced ten times if the AF duration was $\leq 20 \mathrm{~min}$ and five times if the AF lasted between 20 and $30 \mathrm{~min}$. AF that lasted $>30$ min was considered to be persistent, and the mean AF duration was counted as $30 \mathrm{~min}$.

\subsection{Quantification of Fibrosis}

After completion of the electrophysiological study, the heart was rapidly removed and weighed, and the heart to body weight ratio was calculated. The hearts were immersed in a $10 \%$ buffered formalin solution and then embedded in paraffin. To identify possible interstitial fibrosis, Masson-Trichrome staining was carried out in a NexES Special Stainer (Ventana Medical Systems, Tucson, AR, USA). The amount of fibrosis in the LA, which was expressed as a percentage of perimuscular interstitial blue area except muscle for each rat, was quantified with the acquisition software Image-Pro Plus version 5.1 (Media Cybernetics, Maryland, GA, USA).

\subsection{Western Blot}

About $25 \mathrm{mg}$ of rat LA tissue were homogenized in $1 \mathrm{~mL}$ of a modified tonic sucrose solution containing $0.3 \mathrm{~mol} / \mathrm{L}$ sucrose, $10 \mathrm{mmol} / \mathrm{L}$ imidazole, $10 \mathrm{mmol} / \mathrm{L}$ sodium metabisulfite, $1 \mathrm{mmol} / \mathrm{L}$ DTT and $0.3 \mathrm{mmol} / \mathrm{L}$ PMSF. After centrifugation at $1300 \times \mathrm{g}, 50 \mu \mathrm{g}$ of protein for the iNOS and $\mathrm{Na}^{+}-\mathrm{Ca}^{2+}$ exchanger (INCX), thrombomodulin (TM), tissue factor pathway inhibitor (TFPI), tissue plasminogen activator (tPA) and $25 \mu \mathrm{g}$ of protein for the eNOS and SERCA were loaded into 10\% SDS-polyacrylamide gel electrophoresis gel and transferred to a PVDF membrane (Pierce, Rockford, IL, USA). The 
blocked membranes were then incubated with the antibodies to eNOS, iNOS (BD Transduction Laboratories, San Jose, CA, USA), TFPI, TM (American Diagnostica Inc., Stamford, CT, USA) and NCX (Affinity BioReagents, Golden, CO, USA). Horseradish peroxidase (HRP)-conjugated IgG antibody (Santa Cruz Biotechnology, Santa Cruz, CA, USA) was used as the secondary antibody. The immunoblot was visualized using chemiluminescent reagent as recommended by the SuperSignal West Pico Chemiluminescent Substrate kit (Pierce, Rockford, IL, USA). As an internal control, this membrane was re-blotted with anti-GAPDH antibody.

\subsection{Rac1 GST-p21-Activated Kinase Pull-Down Assay}

Tissue was homogenized and resuspended in magnesium-containing lysis buffer $(25 \mathrm{mmol} / \mathrm{L}$ HEPES (pH 7.5); 150 mmol/L sodium chloride; 1\% Igepal CA-630; 10\% glycerol; 25 mmol/L sodium fluoride; $10 \mathrm{mmol} / \mathrm{L}$ magnesium chloride; $1 \mathrm{mmol} / \mathrm{L}$ EDTA; $1 \mathrm{mmol} / \mathrm{L}$ sodium orthovanadate; $10 \mu \mathrm{g} / \mathrm{mL}$ leupeptin; $10 \mu \mathrm{g} / \mathrm{mL}$ aprotinin) and centrifuged at $1000 \mathrm{rpm}$ for $5 \mathrm{~min}$ at $4{ }^{\circ} \mathrm{C}$. Equal amounts of supernatant protein were incubated with $10 \mu \mathrm{L}$ of agarose-labeled p21-activated kinase (PAK)-1 fusion protein at $4{ }^{\circ} \mathrm{C}$ for $60 \mathrm{~min}$. Beads were washed 3-times with magnesium-containing lysis buffer, eluted in Laemmli buffer $(60 \mathrm{mmol} / \mathrm{L}$ Tris $(\mathrm{pH} 6.8), 2 \%$ sodium dodecyl sulfate, $10 \%$ glycerin, $0.1 \%$ bromophenol blue) and analyzed for bound Rac1 in relation to total Rac1 content by western blotting.

\subsection{Immunohistochemistry for eNOS and iNOS}

Sections of left atrial tissue were immunostained with eNOS and iNOS antibody using a horseradish peroxidase-streptavidin-biotin method (UltraVision LP Detection System, Lab Vision Corporation, Suffolk, UK) according to the manufacturer's recommendation. Briefly, formalin-fixed, paraffin-embedded tissue was prepared using conventional histological methods. Serial sections $(6 \mu \mathrm{m})$ were cut from each paraffin block. Samples were immersed in $10 \%$ neutral buffered formalin for $30 \mathrm{~min}$, permeabilized with $0.1 \%$ Triton X-100 for $15 \mathrm{~min}$, and antigen retrieval was achieved by heating in $0.01 \mathrm{M}$ citric acid, pH 6.0, for $20 \mathrm{~min}$. Nonspecific binding was blocked with normal goat serum for 15 minutes. The sections were incubated with anti-eNOS antibody (Santa Cruz, CA, USA) or anti-iNOS antibody (Santa Cruz, CA, USA) at a 1:50 dilution in PBS. After washing with PBS twice for $5 \mathrm{~min}$ each time, incubation was performed for $1 \mathrm{~h}$ at room temperature with biotinylated rabbit anti-rat IgG (1:50) in PBS. Next, the slides were incubated with streptavidin-peroxidase conjugate for $15 \mathrm{~min}$. Peroxidase was visualized by the addition of 3-amino-9-ethyl carbazole (ACE) and hydrogen peroxide. Negative controls were carried out under the same conditions using rat IgG instead of eNOS or iNOS antibody.

\subsection{Statistical Analysis}

We used the resource equation method [40], because it was not possible to assume the effect size, to get an idea about the standard deviation, as no previous findings are available and multiple endpoints are measured. According to this method, a value E (total number of animals - total number of groups) is measured, which is nothing but the degree of freedom of analysis of variance (ANOVA). 
Any sample size that keeps $E$ between 10 and 20 should be considered as adequate. Because we want to see the effect of simvastatin, we made three groups with 10 rats each considering measurement failure, and $E$ was 27 , which is more than necessary. The statistical analysis for each parameter studied was carried out with ANOVA. If the ANOVA tests were significant, inter-group comparisons were performed using the Mann-Whitney test. $p$-values less than 0.05 were considered to be statistically significant. All results are expressed as the mean \pm standard deviation (SD). For the statistical analysis, SPSS version 12.0 (SPSS Inc., Chicago, IL, USA) was used.

\section{Conclusions}

Our observation suggests that the small G protein Rac1 GTPase is upregulated in the ischemic HF model with reduced eNOS, activation of endothelial thromboprotective markers, induction of fibrosis, and increased susceptibility for of AF induction. Simvastatin treatment, which reduces Rac1 activity, was associated with a reduced inducibility of AF, LA fibrosis and thrombogenicity. These results provide a simvastatin-preventing effect of AF in ischemic HF in rats and may be an interesting new approach for preventing arrhythmias associated with ischemic HF-induced LA remodeling in humans.

A limitation of the study is that the model of AF in ischemic HF was induced by pacing. However, classical cardiovascular risk factors influence the genesis, perpetuation, oxidative stress and atherothromboembolism of AF in humans [41]. The dosage of the simvastatin in our study ( $2 \mathrm{mg} / \mathrm{kg} /$ day) was equal to that used in some experimental studies and smaller than that in others, but it was somewhat higher than that used commonly for hypercholesterolemia $(0.3$ to $1 \mathrm{mg} / \mathrm{kg})$ [25]. It still remains to be determined whether clinically-used doses of simvastatin are able to prevent HF-induced AF in humans.

\section{Author Contributions}

T.-J.C. designed the study. S.-H.K. and G.-B.J. directed its implementation, including the study's analytic strategy. J.-H.H., H.-S.K. and J.-W.L. helped conduct the literature review, and K.-I.C. prepared the Methods and the Discussion sections of the text.

\section{Conflicts of Interest}

The authors declare no conflict of interest.

\section{References}

1. Go, A.S.; Hylek, E.M.; Phillips, K.A.; Chang, Y.; Henault, L.E.; Selby, J.V.; Singer D.E. Prevalence of diagnosed atrial fibrillation in adults: National implications for rhythm management and stroke prevention: The AnTicoagulation and Risk Factors in Atrial Fibrillation (ATRIA) Study. JAMA 2001, 285, 2370-2375.

2. Ock, S.Y.; Cho, K.I.; Kim, H.J.; Lee, N.Y.; Kim, E.J.; Kim, N.K.; Lee, W.H.; Yeo, G.E.; Heo, J.J.; Han, Y.J.; et al. The impacts of C-reactive protein and atrial fibrillation on carotid atherosclerosis and ischemic stroke in patients with suspected ischemic cerebrovascular disease: A single-center retrospective observational cohort study. Korean Circ. J. 2013, 43, 796-803. 
3. Korantzopoulos, P.; Kolettis, T.; Siogas, K.; Goudevenos, J. Atrial fibrillation and electrical remodeling: The potential role of inflammation and oxidative stress. Med. Sci. Monit. 2003, 9, RA225-RA229.

4. Cai, H.; Li, Z.; Goette, A.; Mera, F.; Honeycutt, C.; Feterik, K.; Wilcox, J.N.; Dudley, S.C.; Harrison, D.G.; Langberg, J.J. Down-regulation of endocardial nitric oxide synthase expression and nitric oxide production in atrial fibrillation: Potential mechanisms for atrial thrombosis and stroke. Circulation 2002, 106, 2854-2858.

5. Aviles, R.J.; Martin, D.O.; Apperson-Hansen, C.; Houghtaling, P.L.; Rautaharju, P.; Kronmal, R.A.; Tracy, R.P.; van Wagoner, D.R.; Psaty, B.M.; Lauer, M.S.; et al. Inflammation as a risk factor for atrial fibrillation. Circulation 2003, 108, 3006-3010.

6. Maron, D.J.; Fazio, S.; Linton, M.F. Current perspectives on statins. Circulation 2000, 101, 207-213.

7. Ludmer, P.L.; Selwyn, A.P.; Shook, T.L.; Wayne, R.R.; Mudge, G.H.; Alexander, R.W.; Ganz, P. Paradoxical vasoconstriction induced by acetylcholine in atherosclerotic coronary arteries. N. Engl. J. Med. 1986, 315, 1046-1051.

8. Laufs, U.; Liao, J.K. Post-transcriptional regulation of endothelial nitric oxide synthase mRNA stability by rho gtpase. J. Biol. Chem. 1998, 273, 24266-24271.

9. Maguy, A.; Hebert, T.E.; Nattel, S. Involvement of lipid rafts and caveolae in cardiac ion channel function. Cardiovasc. Res. 2006, 69, 798-807.

10. Feron, O.; Balligand, J.L. Caveolins and the regulation of endothelial nitric oxide synthase in the heart. Cardiovasc. Res. 2006, 69, 788-797.

11. Pound, E.M.; Kang, J.X.; Leaf, A. Partitioning of polyunsaturated fatty acids, which prevent cardiac arrhythmias, into phospholipid cell membranes. J. Lipid Res. 2001, 42, 346-351.

12. Ganotakis, E.S.; Mikhailidis, D.P.; Vardas, P.E. Atrial fibrillation, inflammation and statins. Helle. J. Cardiol. 2006, 47, 51-53.

13. Young-Xu, Y.; Jabbour, S.; Goldberg, R.; Blatt, C.M.; Graboys, T.; Bilchik, B.; Ravid, S. Usefulness of statin drugs in protecting against atrial fibrillation in patients with coronary artery disease. Am. J. Cardiol. 2003, 92, 1379-1383.

14. Dernellis, J.; Panaretou, M. C-reactive protein and paroxysmal atrial fibrillation: Evidence of the implication of an inflammatory process in paroxysmal atrial fibrillation. Acta Cardiol. 2001, 56, 375-380.

15. Sarr, F.S.; André, C.; Guillaume, Y.C. Statins (HMG-coenzyme A reductase inhibitors)-biomimetic membrane binding mechanism investigated by molecular chromatography J. Chromatogr. B 2008, 868, 20-27.

16. Davidson, M.H.; Toth, P.P. Comparative effects of lipid-lowering therapies. Prog. Cardiovasc. Dis. 2004, 47, 73-104.

17. Antoons. G.; Oros, A.; Bito, V.; Sipido, K.R.; Vos, M.A. Cellular basis for triggered ventricular arrhythmias that occur in the setting of compensated hypertrophy and heart failure: Considerations for diagnosis and treatment. J. Electrocardiol. 2007, 40, S8-S14.

18. Mørk, H.K.; Sjaastad, I.; Sande, J.B.; Periasamy, M.; Sejersted, O.M.; Louch W.E. Increased cardiomyocyte function and $\mathrm{Ca}^{2+}$ transients in mice during early congestive heart failure. J. Mol. Cell. Cardiol. 2007, 43, 177-186. 
19. Del Monte, F.; Lebeche, D.; Guerrero, J.L.; Tsuji, T.; Doye, A.A.; Gwathmey, J.K.; Hajjar, R.J. Abrogation of ventricular arrhythmias in a model of ischemia and reperfusion by targeting myocardial calcium cycling. PNAS 2004, 101, 5622-5627.

20. Satoh, M.; Ogita, H.; Takeshita, K.; Mukai, Y.; Kwiatkowski, D.J.; Liao, J.K. Requirement of Rac1 in the development of cardiac hypertrophy. Proc. Natl. Acad. Sci. USA 2006, 103, 7432-7437.

21. Adam, O.; Frost, G.; Custodis, F.; Sussman, M.A.; Schäfers, H.J.; Böhm, M.; Laufs, U. Role of Rac1 GTPase activation in atrial fibrillation. J. Am. Coll. Cardiol. 2007, 50, 359-367.

22. Cha, T.J.; Ehrlich, J.R.; Zhang, L.; Shi, Y.F.; Tardif, J.C.; Leung, T.K.; Nattel, S. Dissociation between ionic remodeling and ability to sustain atrial fibrillation during recovery from experimental congestive heart failure. Circulation 2004, 109, 412-418.

23. Patel, R.; Nagueh, S.F.; Tsybouleva, N.; Abdellatif, M.; Lutucuta, S.; Kopelen, H.A.; Quinones, M.A.; Zoghbi, W.A.; Entman, M.L.; Roberts, R.; et al. Simvastatin induces regression of cardiac hypertrophy and fibrosis and improves cardiac function in a transgenic rabbit model of human hypertrophic cardiomyopathy. Circulation 2001, 104, 317-324.

24. Stmbler, B.S.; Fenelon, G.; Shepard, R.K.; Clemo, H.F.; Guiraudon, C.M. Characterization of sustained atrial tachycardia in dogs with rapid ventricular pacing-induced heart failure. J. Cardiovasc. Electrophysiol. 2003, 14, 499-507.

25. Shiroshita-Takeshita, A.; Schram, G.; Lavoie, J.; Nattel, S. Effect of simvastatin and antioxidant vitamins on atrial fibrillation promotion by atrial-tachycardia remodeling in dogs. Circulation 2004, 110, 2313-2319.

26. Miyano, K.; Sumimoto, H. Role of the small GTPase Rac in $\mathrm{p} 22^{\text {phox }}$-dependent NADPH oxidases. Biochimie 2007, 89, 1133-1144.

27. Mizuno, T.; Kaibuchi, K.; Ando, S.; Musha, T.; Hiraoka, K.; Takaishi, K.; Asada, M.; Nunoi, H.; Matsuda, I.; Takai, Y. Regulation of the superoxide-generating NADPH oxidase by a small GTP-binding protein and its stimulatory and inhibitory GDP/GTP exchange proteins. J. Biol. Chem. 1992, 267, 10215-10218.

28. Cangemi, R.; Celestini, A.; Calvieri, C.; Carnevale, R.; Pastori, D.; Nocella, C.; Vicario, T.; Pignatelli, P.; Violi, F. Different behaviour of NOX2 activation in patients with paroxysmal/persistent or permanent atrial fibrillation. Heart 2012, 98, 1063-1066.

29. Laufs, U.; Kilter, H.; Konkol, C.; Wassmann, S.; Böhm, M.; Nickenig, G. Impact of HMG CoA reductase inhibition on small GTPases in the heart. Cardiovasc. Res. 2002, 3, 911-920.

30. Svetlana, N.R.; Raja, J.; Keshav, N.; Charalambos, A.; Sander, V.; Keith, M.C.; Nicholas, J.A.; Ulrich S.; Barbara C. Atrial sources of reactive oxygen species vary with the duration and substrate of atrial fibrillation: Implications for the antiarrhythmic effect of statins. Circulation 2011, 124, 1107-1117.

31. Fukuchi, M.; Hussain, S.N.; Giaid, A. Heterogeneous expression and activity of endothelial and inducible nitric oxide synthases in end-stage human heart failure: their relation to lesion site and beta-adrenergic receptor therapy. Circulation 1998, 98, 132-139.

32. Fischmeister, R.; Castro, L.; Abi-Gerges, A.; Rochais, F.; Vandecasteele, G. Species- and tissue-dependent effects of NO and cyclic GMP on cardiac ion channels. Comp. Biochem. Physiol. A 2005, 142, 136-143. 
33. Massion, P.B.; Feron, O.; Dessy, C.; Balligand, J.L. Nitric oxide and cardiac function: Ten years after, and continuing. Circ. Res. 2003, 93, 388-398.

34. Damy, T.; Ratajczak, P.; Shah, A.M.; Camors, E.; Marty, I.; Hasenfuss, G.; Marotte, F.; Samuel, J.L.; Heymes, C. Increased neuronal nitric oxide synthase-derived NO production in the failing human heart. Lancet 2004, 363, 1365-1367.

35. Kim, Y.M.; Guzik, T.J.; Zhang, Y.H.; Zhang, M.H.; Kattach, H.; Ratnatunga, C.; Pillai, R.; Channon, K.M.; Casadei, B. A myocardial NOX2 containing NADPH oxidase contributes to oxidative stress in human atrial fibrillation. Circ. Res. 2005, 97, 629-636.

36. Dudley, S.C., Jr.; Hoch, N.E.; McCann, L.A., Honeycutt, C.; Diamandopoulos, L.; Fukai, T.; Harrison, D.G.; Dikalov, S.I.; Langberg, J. Atrial fibrillation increases production of superoxide by the left atrium and left atrial appendage: Role of the NADPH and xanthine oxidases. Circulation 2005, 112, 1266-1273.

37. Kim, H.S.; No, C.W.; Goo, S.H.; Cha, T.J. An angiotensin receptor blocker prevents arrhythmogenic left atrial remodeling in a rat post myocardial infarction induced heart failure model. J. Korean Med. Sci. 2013, 28, 700-708.

38. Pogwizd, S.M.; Schlotthauer, K.; Li, L.; Yuan, W.; Bers, D.M. Arrhythmogenesis and contractile dysfunction in heart failure: Roles of sodium-calcium exchange, inward rectifier potassium current, and residual $\beta$-adrenergic responsiveness. Circ. Res. 2001, 88, 1159-1167.

39. Bers, D.M. Cardiac excitation-contraction coupling. Nature 2002, 415, 198-205.

40. Festing, M.F.; Altman, D.G. Guidelines for the design and statistical analysis of experiments using laboratory animals ILAR J. 2002, 43, 244-228.

41. Violi, F.; Loffredo, L. Thromboembolism or atherothromboembolism in atrial fibrillation? Circ. Arrhythm. Electrophysiol. 2012, 5, 1053-1055.

(C) 2014 by the authors; licensee MDPI, Basel, Switzerland. This article is an open access article distributed under the terms and conditions of the Creative Commons Attribution license (http://creativecommons.org/licenses/by/3.0/). 